\title{
Keratin 17 is a sensitive and specific biomarker of urothelial neoplasia
}

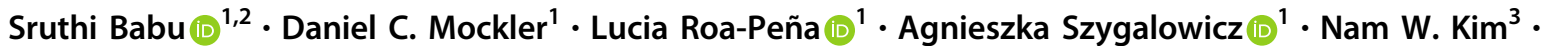 \\ Sholeh Jahanfard ${ }^{3} \cdot$ Shahram S. Gholami ${ }^{3} \cdot$ Richard Moffitt $^{1,4} \cdot$ John P. Fitzgerald $^{5} \cdot$ Luisa F. Escobar-Hoyos $^{1,6,7}$. \\ Kenneth R. Shroyer ${ }^{1}$
}

Received: 18 June 2018 / Revised: 18 October 2018 / Accepted: 26 October 2018 / Published online: 15 November 2018

(c) United States \& Canadian Academy of Pathology 2018

\begin{abstract}
There is a clinical need to identify novel biomarkers to improve diagnostic accuracy for the detection of urothelial tumors. The current study aimed to evaluate keratin 17 (K17), an oncoprotein that drives cell cycle progression in cancers of multiple anatomic sites, as a diagnostic biomarker of urothelial neoplasia in bladder biopsies and in urine cytology specimens. We evaluated K17 expression by immunohistochemistry in formalin-fixed, paraffin embedded tissue specimens of non-papillary invasive urothelial carcinoma (UC) (classical histological cases), high grade papillary UC (PUC-LG), low grade papillary UC (PUC-HG), papillary urothelial neoplasia of low malignant potential (PUNLMP), and normal bladder mucosa. A threshold was established to dichotomize K17 status in tissue specimens as positive vs. negative, based on the proportion of cells that showed strong staining. In addition, K17 immunocytochemistry was performed on urine cytology slides, scoring positive test results based on the detection of K17 in any urothelial cells. Mann-Whitney and receiver operating characteristic analyses were used to compare K17 expression between histologic diagnostic categories. The median proportion of K17 positive tumor cells was 70\% (range 20-90\%) in PUNLMP, 30\% (range 5-100\%) in PUC-LG, 20\% (range 1-100\%), in PUC-HG, 35\% (range 5-100\%) in UC but staining was rarely detected (range 0-10\%) in normal urothelial mucosa. Defining cases in which K17 was detected in $\geq 10 \%$ of cells were considered positive, the sensitivity of K17 in biopsies was 89\% (95\% CI: 80-96\%) and the specificity was 88\% (95\% CI: 70-95\%) to distinguish malignant lesions (PUC-LG, PUC-HG, and UC) from normal urothelial mucosa. Furthermore, K17 immunocytochemistry had a sensitivity of $100 \%$ and a specificity of $96 \%$ for urothelial carcinoma in 112 selected urine specimens. Thus, K17 is a sensitive and specific biomarker of urothelial neoplasia in tissue specimens and should be further explored as a novel biomarker for the cytologic diagnosis of urine specimens.
\end{abstract}

These author contributed equally: Luisa F. Escobar-Hoyos, Kenneth R. Shroyer

Electronic supplementary material The online version of this article (https://doi.org/10.1038/s41379-018-0177-5) contains supplementary material, which is available to authorized users.

Luisa F. Escobar-Hoyos

luisa.escobarhoyos@stonybrookmedicine.edu

$\triangle$ Kenneth R. Shroyer

Kenneth.Shroyer@stonybrookmedicine.edu

1 Department of Pathology, Stony Brook Medicine, Stony Brook, NY, USA

2 Program of Public Health and Department of Preventative Medicine, Stony Brook Medicine, Stony Brook, NY, USA

3 KDx Diagnostics Inc, Campbell, CA, USA

\section{Introduction}

Bladder cancer is the second most common cancer of the urinary system and accounts for about 81,190 new cases and 17,240 cancer related deaths each year in the United

4 Department of Biomedical Informatics, Stony Brook Medicine, Stony Brook, NY, USA

5 Department of Urology, Stony Brook Medicine, Stony Brook, NY, USA

6 David M. Rubenstein Center for Pancreatic Cancer Research, Memorial Sloan Kettering Cancer Center, New York, New York 10065, USA

7 Department of Biology, Genetic Toxicology and Cytogenetics Research Group, School of Natural Sciences and Education, Universidad Del Cauca, Popayán, Colombia 
States [1]. Even though the majority of cases are diagnosed at early stage, the recurrence rate is very high, necessitating frequent surveillance, contributing to high cost for the treatment and follow-up of bladder cancer patients [1-3]. The histologic assessment of cystoscopic biopsy specimens can also be challenging, due to small sample size, suboptimal orientation, erosion of surface epithelium, or sampling and processing artifacts that can lead to false negative or false positive diagnoses. Urine cytology is the most common diagnostic test used for both screening and surveillance of bladder cancer but the sensitivity of this test is very low for low grade lesions [4]. Furthermore, diagnostic accuracy of urine cytology specimens can be impacted by cellularity, methods of collection, and processing artifacts but also by the absence of definitive cytologic manifestations of malignancy in low-grade urothelial carcinomas. Although a variety of FDA-approved tests for urothelial cancer provide enhanced diagnostic accuracy, those that focus on the detection of aneuploidy may be negative in low grade urothelial carcinomas while those that are focused on the detection of tumor-associated antigens may show positive test results in the absence of neoplasia [4-14]. Thus, there remains an unmet need to identify biomarkers of urothelial carcinoma that could be deployed to enhance diagnostic accuracy in both bladder biopsy and urine cytology specimens.

We previously used mass spectrometry to identify K17 as a prognostic biomarker in cervical squamous cell carcinoma [15] and our loss- and gain-of-function studies subsequently led to the discovery that K17 targets p27, a tumor suppressor protein, for nuclear export, to promote sustained cell cycle progression [16]. Others studies show that K17 is a negative prognostic marker in endocervical adenocarcinoma [17], triple negative breast cancer [18], head and neck squamous cell carcinoma [19], gastric adenocarcinoma [20], and ovarian cancer [21].

By bioinformatic analyses of a publically available gene expression dataset [22], we compared the expression of $\mathrm{K} 17$ in cancers of different anatomic sites to expression in normal tissue. While K17 levels of expression were highly variable across cancers of many other anatomic sites, K17 was expressed in $100 \%$ of urothelial carcinomas at a level 2.5 to $8 \log$ fold greater than in normal urothelial mucosa (Fig. 1). This finding strongly supports our hypothesis that K17 could be a diagnostic biomarker for bladder cancer compared to other tumors.

Thus, the aims of the current study were to explore the accuracy of $\mathrm{K} 17$ as a potential diagnostic biomarker in bladder tissue specimens and to further determine if K17 could also detect urothelial cancer in urine cytology specimens.

\section{Methods}

\section{Validation of K17 as a biomarker in tissue specimens}

\section{Case selection}

A total of 109 formalin-fixed paraffin-embedded surgical tissue blocks, from 109 discrete patients that received care at Stony Brook Medicine from 1995-2017, were retrospectively selected from the archival collections of the Stony Brook BioBank, in compliance with IRB-approved protocols (CORIHS Protocol \#94651). These cases included benign urothelium, $(n=25)$, papillary urothelial lesion of low malignant potential (PUNLMP, $n=9$ ), low-grade papillary urothelial carcinoma (PUC-LG, $n=25$ ), highgrade papillary urothelial carcinoma (PUC-HC, encompassing both non-invasive and invasive lesions, $n=24)$, and non-papillary, invasive urothelial carcinoma (UC, $n=$ 26) (Fig. 2). Less common histologic types (micropapillary

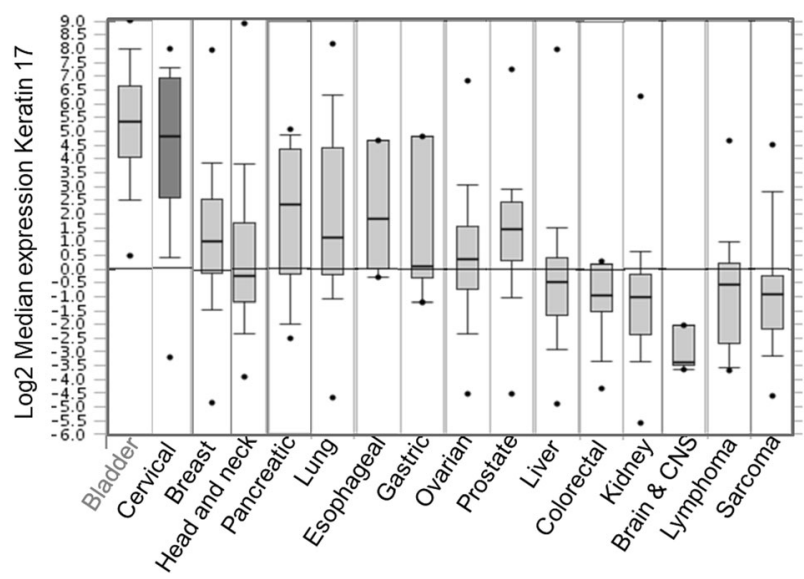

Fig. 1 Keratin 17 is differentially expressed in urothelial cancers relative to normal tissue. The $Y$-axis shows the log fold changes of K17 expression compared to normal tissue expression. K17 expression above the $\log$ zero represent increased expression

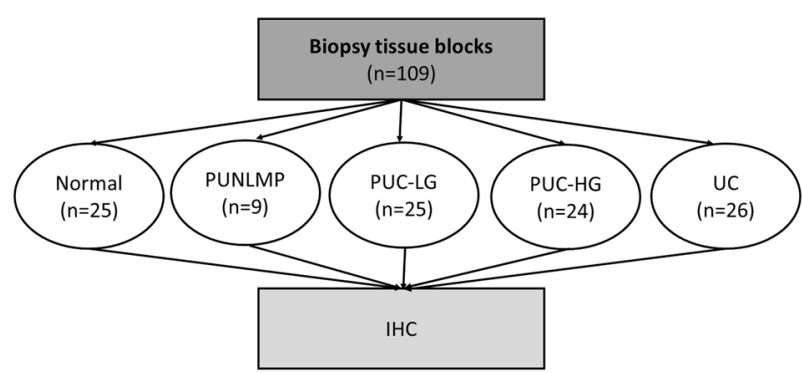

Fig. 2 Flow chart, validation and quantification of Keratin 17 in tissue. Normal: Benign urothelial mucosa, PUC-LG Papillary Urothelial Carcinoma-Low Grade, PUC-HG Papillary Urothelial CarcinomaHigh Grade, UC Non-papillary Urothelial Carcinoma, IHC Immunohistochemistry 
urothelial carcinomas, nested urothelial carcinomas, squamous cell carcinomas, adenocarcinomas, or carcinomas that were metastatic to the bladder), however, were not included in the study. In all cases, a tissue block was selected following histologic review of hematoxylin and eosin stained sections from bladder mucosal biopsies $(n=86)$, transurethral resections of bladder tumors (TURBT) $(n=2)$, or cystectomy specimens $(n=21)$ to confirm that diagnostic tissue was represented in the residual tissue blocks.

Immunohistochemical localization of K17 was performed as previously described $[15,17]$. Briefly, formalinfixed, paraffin-embedded tissue blocks were sectioned, mounted on charged glass slides and deparaffinized. Antigen retrieval was performed using a decloaking chamber at $120^{\circ} \mathrm{C}$ for $10 \mathrm{~min}$ in citrate buffer $(20 \mathrm{mmol}, \mathrm{pH} 6.0$, Vector Laboratories, Burlingame, CA, USA). Sections were incubated overnight with mouse monoclonal anti-human K17 antibody (clone E3 from both Thermo Fisher Scientific, catalog \# MA5-13539, Grand Island, NY USA and KDX, lot: A8034124, Campbell, CA USA) and negative controls were performed using an equivalent concentration of a subclass matched mouse immunoglobulin, generated against unrelated antigens (BD, Franklin Lakes, NJ), in place of primary antibody. Following incubation with the primary antibody, slides were processed by an indirect avidin-biotin based immunoperoxidase method (R.T.U. Vectastain Universal Elite ABC kit, Vector Laboratories, Burlingame, CA, USA), developed in 3,3' diaminobenzidine (DAB) (DAKO, Carpentaria, CA, USA) and counterstained with hematoxylin. K17 staining was recorded using a manual semi-quantitative scoring system that is based on the proportion of tumor cells with strong staining (1-100\%, Path SQ Score). Immunohistochemical scoring of K17 expression in biopsy specimens was performed independently by two surgical pathologists (D.M and K.R.S). No discordance was observed in the final classification of K17 status (Path SQ Score $<10$ vs. Path SQ score $\geq 10$ ).

\section{Validation of K17 as a biomarker in urine cytology}

\section{Case selection}

A total of 69 remnant ThinPrep CytoLyt-fixed urine specimens were collected between 2016-2018 by the Stony Brook University Hospital Cytology and 43 urine specimens were collected by a community-based urologic clinic (Urology associates of Silicon Valley), in compliance with IRB-approved protocols (CORIHS protocols 94651,Stony Brook University and 00025188, KDx Diagnostics Inc.) (Fig. 3). The 69 urine samples from Stony Brook Medicine included 25 cases with a biopsy-
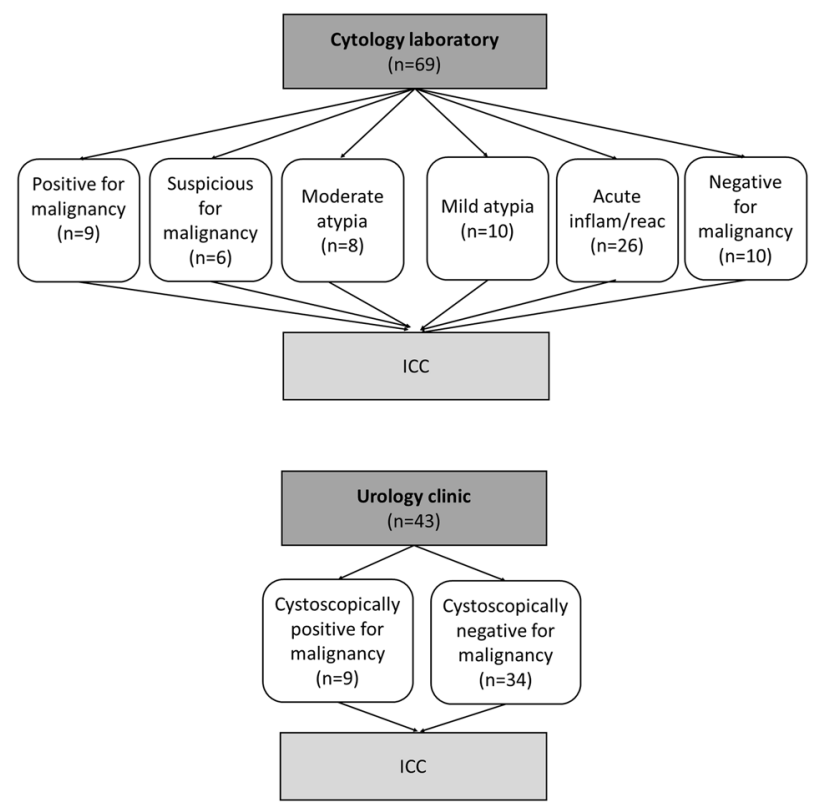

Fig. 3 Flow chart, validation and quantification of Keratin 17 in urine specimens. The upper panel represents remnant urine specimens from the Stony Brook Medicine Cytology Laboratory. The lower panel represents urine specimens collected by a community practice urology clinic, with final diagnosis based on cystoscopic diagnosis with biopsy confirmation. ICC Immunocytochemistry

confirmed diagnosis of urothelial carcinoma. Of those cases, the cytologic diagnosis was positive for malignancy $(n=9)$, suspicious for malignancy $(n=6)$, moderate atypia $(n=5)$, mild atypia $(n=4)$, or reactive changes $(n=1)$. The 44 urine cytology samples that had no biopsy evidence of urothelial carcinoma had a cytologic diagnosis of moderate atypia $(n=3)$, mild atypia $(n=6)$, acute inflammation with reactive changes $(n=25)$, or negative for malignancy $(n=10)$ (S-Table 1$)$. Urine cytology data was not available for samples from the community practice urologic clinic $(n=43)$. Of these cases, final diagnosis was based on cystoscopic findings with biopsy, including 9 cases with a final diagnosis of urothelial carcinoma and 34 that were negative for carcinoma (Fig. 3).

\section{Urine Immunocytochemistry (ICC)}

Urine samples, collected in PreservCyt (Hologic, Marlborough, MA), were centrifuged at $1000 \times g$ for $10 \mathrm{~min}$, the pelleted cells were resuspended in $20 \mathrm{~mL}$ of PreservCyt, and were transferred onto changed glass slides using a Thinprep 2000 (Hologic) processor. Endogenous peroxidase activity was blocked using BloxAll (Vector Laboratories, Burlingame, CA) and non-specific antibody binding was blocked by pre-incubation with $2.5 \%$ horse serum (Vector Laboratories, Burlingame, CA). Following incubation with anti-K17 antibody, as described above, slides were 
Table 1 Urine specimens: Keratin 17 immunocytochemistry has a higher sensitivity and specificity than cytologic examination for urothelial carcinoma

\begin{tabular}{lll}
\hline Test & Cytology $^{\mathrm{a}} \%(95 \% \mathrm{CI})$ & K17 ICC $^{\mathrm{b}} \%(95 \% \mathrm{CI})$ \\
\hline Sensitivity $^{\mathrm{c}}$ & $80(61-91)$ & $100(90-100)$ \\
Specificity $^{\mathrm{c}}$ & $93(82-98)$ & $96(89-99)$ \\
PPV & $87(68-95)$ & $91(79-97)$ \\
NPV & $89(77-95)$ & $100(95-100)$ \\
\hline
\end{tabular}

$C I$ confidence Interval, $P P V$ positive predictive value, $N P V$ negative predictive value

${ }^{a}$ Urine cytology diagnoses based on samples from Stony Brook Medicine

Negative cytology test results include cases with a diagnosis of no evidence of malignancy, reactive changes, acute inflammation, and/or mild atypia. Positive urine cytology test results included cases scored as moderate atypia, severe atypia/suspicious for carcinoma, and positive for carcinoma

${ }^{b}$ K17 immunocytochemistry results based on samples from both Stony Brook Medicine and a community-based urologic clinic

${ }^{\mathrm{c}}$ Sensitivity and specificity were calculated based on biopsy confirmation of lesions

processed by a direct polymer-based immunoperoxidase method (MediaView Two-Step Anti-mouse \& rabbit polymer-HRP system, Medaysis, Alameda, CA), developed in $\mathrm{DAB}$, and counterstained with hematoxylin. All immunocytochemical stains were reviewed by single cytopathologist (K.R.S) to score K17 test results, based on the detection of staining in urothelial cells, regardless of the presence or absence of cytologic atypia.

\section{Statistical analysis}

Mann-Whitney and receiver operating curves analyses were used to compare K17 expression in non-neoplastic vs. neoplastic tissue specimens and ROC and the area under the curve [23] were calculated from Path SQ Score to evaluate the biomarker potential to discriminate different diagnostic categories. The optimal cutoff value from ROC was determined using Youden's index [24], which corresponded to a Path SQ Score $\geq 10$. Sensitivity and specificity were calculated, corresponding to the optimal cutoff values. The sample sensitivity and specificity of urine K17 immunocytochemistry for detection of biopsy-confirmed urothelial carcinoma was calculated by comparison with a concurrent or prior histologic diagnosis of urothelial carcinoma. Samples that had no history of abnormal urine cytology or tissue diagnosis of carcinoma were categorized as negative for urothelial carcinoma. Statistical significance was set at $p$-value $<0.05$ and analyses were performed using SAS 9.4 (SAS Institute, Cary, NC, USA) and Graph pad Prism 7 (Graph Pad Software, La Jolla, CA, USA).

\section{Results}

\section{K17 is a diagnostic biomarker in urothelial tissue}

Keratin 17 was detected in approximately $5 \%$ of basal urothelial cells in 2 biopsies and in about $10 \%$ of basal urothelial cells in one biopsy of normal bladder mucosa. The remaining 22 benign bladder mucosal biopsy specimens showed no staining for K17. By contrast, K17 was detected in $30 \%$ of tumor cells in PUC-LG (range 5-100\%), in $20 \%$ of tumor cells in PUC-HG (range 1-100\%), and in $35 \%$ of tumor cells in UC (range 5-100\%) (Fig. 4a, b). Non-papillary urothelial carcinomas (UC) had higher expression of K17 than papillary carcinomas (PUC-LG plus PUC-HG) $\quad(p$-value $<0.05$ ) (Supplementary Figure 1A). Furthermore, K17 was detected in a higher proportion of tumor cells in muscle invasive UC (5-100\%, median 40\%) than in non-muscle invasive UC (1-100\%, median $20 \%)$ and the differences were statically significantly significant $(p<0.05$, Supplementary Figure 1B). K17 was also detected in 9/9 PUNLMPs (scores ranged from 20-90\%, median $70 \%$ ) but was rarely detected in basal urothelial cells of normal or reactive bladder mucosa. Across all diagnostic categories, the difference in the proportion of cells that stained for $\mathrm{K} 17$ in non-neoplastic mucosa vs. urothelial carcinomas was significant $(\mathrm{ROC}$ AUC $=0.96, p<0.001$ ) and was unrelated to patient age and gender (data not shown). Setting the threshold to categorize positive vs. negative test results in histologic sections as strong K17 staining in $\geq 10$ of cells, the sensitivity of K17 was 89\% (95\%CI: $80-96 \%$ ) and the specificity was $88 \%$ (95\% CI: 70-95\%) for malignant lesions (PUC-LG, PUC-HG and UC) vs. normal urothelial mucosa (Fig. 4c). Thus, our results suggested that the immunohistochemical detection of K17 in tissue discriminates malignant lesions (PUC-LG, PUC-HG and UC) with both high sensitivity and specificity from normal urothelial mucosa but that positive test results do not exclude the diagnosis of PUNLMP.

\section{$\mathrm{K} 17$ as diagnostic biomarker in urine cytology}

Immunocytochemical studies were performed in 112 urine specimens to determine if the detection of K17 was related to cytologic diagnosis. Cases were scored positive based on the detection of K17 staining in urothelial cells, irrespective of the presence or absence of cytological features that were diagnostic of malignancy. Although strong staining was only detected in urothelial cells, faint cytoplasmic staining was occasionally seen in benign squamous epithelial cells, most commonly in urine specimens from female patients (Fig. 5a-d). Inflammatory cells were consistently negative for K17. At least 20 (20-100, median 30) immunoreactive urothelial cells were detected in slides from cytologically 
A

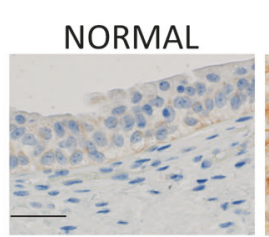

B

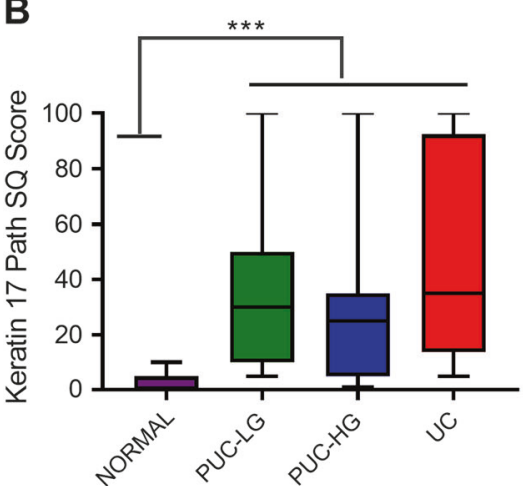

Fig. 4 Immunohistochemical detection of Keratin 17 expression in tissue specimens, representative cases. a Normal: Benign urothelial mucosa; PUC-LG: Papillary Urothelial Carcinoma Low Grade; PUCHG: Papillary Urothelial Carcinoma High Grade; UC: Urothelial Carcinoma. Scale bar; $50 \mu \mathrm{m}$. b Expression of K17 in each diagnostic category, based on the percentage of cells with strong staining. $* * * P<$ $0.001 * * P<0.05$ by Kruskal-Wallis and Wilcoxon rank-sum test. c Keratin17 receiver operating curve analysis and sensitivity and
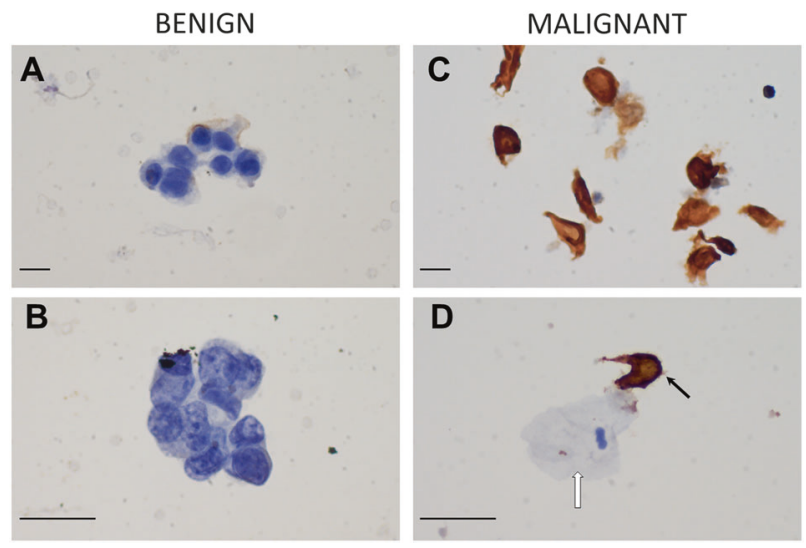

Fig. 5 Detection of Keratin 17 expression in urine cytology specimens, representative cases. a Benign urothelial cells; b Normal Urothelial cells; c K17 positive urothelial cells; d K17 positive urothelial cell (black arrow) adjacent to benign squamous cell (White arrow). Scale bar; $20 \mu \mathrm{m}$ (a and c), $50 \mu \mathrm{m}$ (b and d)

malignant urine specimens that had biopsy confirmed urothelial carcinomas. Among 69 cases for which both cytologic diagnosis and final clinicopathologic diagnosis were available from the Stony Brook Medicine Cytology Laboratory cohort (Fig. 3), K17 was detected in 0/10 cases with negative urine cytology, in $1 / 26(3.8 \%)$ with acute inflammation or benign reactive changes, in $4 / 10(40 \%)$ of cases with mild atypia, and in $100 \%$ of cases classified as moderate atypia
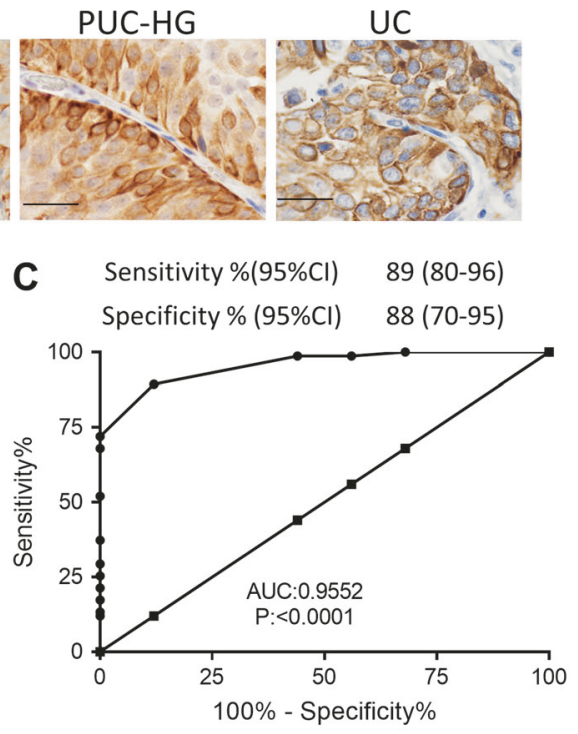

specificity in diagnosing different diagnostic categories of urothelial carcinoma according to Path SQ Score (IHC). AUC Area under the curve, IHC Immunohistochemistry, CI Confidence Interval. The optimal cutoff value from receiver operating curves was determined using Youden's index. The optimal cutoff value in the resultant receiver-operating curve corresponded to $\geq 10 \%$ of positive cells. Sensitivity and specificity were calculated corresponding to the optimal cutoff values

$(n=5)$, suspicious for carcinoma $(n=6)$, or positive for urothelial carcinoma $(n=9)$ (S-Table 1). In this series, biopsy-confirmed urothelial carcinoma was found in $25 / 28$ K17 ICC positive cases. Three urine specimens with moderate cytologic atypia, however, were positive for K17 by ICC but had no current or prior biopsy diagnosis of urothelial carcinoma. Also of interest, one case that had a negative cytological diagnosis but was positive for K17 proved to have high-grade urothelial carcinoma of the renal pelvis.

Among the 43 urine samples from a community practice urologic clinic (Fig. 3), K17 ICC was positive in 9/9 cases that had a cystoscopic diagnosis of urothelial carcinoma but was not detected in 34 specimens from patients that had negative findings on cystoscopy. Thus, relative to the final diagnosis of urothelial carcinoma in 34 biopsy confirmed samples from both a cytology laboratory and a urologic clinic, K17 urine immunocytochemistry had a sensitivity of 100\% (95\% CI: $90-100 \%$ ), a specificity of $96 \%$ (95\% CI: $89-99 \%)$, a positive predictive value of $91 \%(95 \% \mathrm{Cl}$ : $79-97 \%)$, and the negative predictive value of $100 \%(95 \%$ Cl: $95-100 \%$ ) for urothelial carcinoma (Table 1).

\section{Discussion}

$\mathrm{K} 17$ is an embryonic keratin, silenced in most adult somatic tissues except certain stem cell populations and 
re-expressed in a wide range of cancer types [25, 26]. Although K17 is expressed in only a proportion of cancer cases of other anatomic sites (where K17 status correlates with tumor aggression and decreased survival), in bladder cancer, essentially all cases, including all major diagnostic subsets, are K17 test positive, suggesting that in this context, it could serve as a sensitive and specific diagnostic biomarker. The proportion of K17 positive cells, however, was greater in non-papillary UC than in papillary carcinomas and the proportion of tumor cells that expressed K17 was greater in muscle invasive UC compared to non-muscle invasive UCs. K17 was also expressed in a high proportion of cells in PUNLMP, a low grade papillary neoplastic process that is thought to only rarely progress to invasive urothelial carcinoma [27]. Thus, in contrast to prior studies, which have focused on the role of K17 as a prognostic biomarker [15, 17, 18, 28], K17 does not appear to be a consistent biomarker of urothelial tumor aggression. A study performed using a transformed urothelial cancer cell line demonstrated that K17 expression was correlated with foci of squamous differentiation of urothelial tumor cells [29] and another study reported that K17 is highly expressed not only in urothelial tumors but is also focally positive in basal cells of normal appearing urothelium [30]. By contrast, we found K17 was significantly expressed in essentially all urothelial neoplastic lesions tested, but is expressed in few basal cells of normal bladder mucosa. These findings suggest that K17 could be useful as a diagnostic biomarker of urothelial neoplasia.

We further conducted exploratory studies to show that K17 could be detected in urine cytology specimens and that in highly selected cases, including a high proportion of specimens from patients with a diagnosis of urothelial carcinoma, that K17 immunocytochemical staining was detected only in urothelial cancer cases but not in benign urine cytology specimens. In our cases from the Stony Brook Medicine Cytology Laboratory, in which we compared K17 test results to urine cytologic diagnosis, K17 was positive in $100 \%$ of the malignant cases, all of which were confirmed by cystoscopy and subsequent biopsy or tumor resection. K17 was positive in three urine cytology cases with moderate atypia but biopsy data were not available to confirm the diagnosis. All three of these cases had no diagnosis of urothelial carcinoma in the past, thus these cases may represent false positive K17 test results. K17 was also detected in one of the cases that had negative findings, based on urine cytologic diagnosis and on bladder biopsy but proved to have high-grade urothelial carcinoma of the renal pelvis. Thus, K17 immunocytochemistry not only detected urothelial carcinomas of the urinary bladder but also detected a case from the upper urinary tract that was missed by urine cytology. Our study also included a cohort of patient urine specimens from a community practice- based urology clinic. K17 Immunocytochemistry had similar specificity to urine cytology. The sensitivity was higher in K17 Immunocytochemistry compared to urine cytology but was marginally statistically significant in Stony Brook cases. Thus, our findings suggest that K17 could have a role as a screening biomarker or as a diagnostic adjunct to screen for disease recurrence following surgical intervention. Further insight into the performance of $\mathrm{K} 17$ as a marker for primary screening or to monitor disease recurrence, however, will depend on the outcome of larger prospective clinical trials.

Many immunohistochemical markers have been reported to differentiate benign changes from neoplastic processes of the bladder mucosa, including the loss of cytoplasmic CD44 expression, increased keratin 20 expression in deeper layers of the urothelium, the detection of diffuse nuclear staining for p53, and a high Ki-67 index [31-34]. Each of these biomarkers, however, has been shown to have relatively high expression in high tumor grade UC but low sensitivity for low grade urothelial carcinomas. Histopathology has continued to serve as the gold standard for bladder cancer diagnosis and as the most important prognostic factor to predict clinical behavior [35]. The current findings, however, suggest that K17 could serve as a sensitive and specific biomarker to detect PUNLMP and both papillary and non-papillary carcinomas in tissue specimens, potentially providing diagnostic utility in cases where the minute size of the lesion or other factors may challenge accurate diagnostic classification based only on the evaluation of hematoxylin and eosin-stained section.

Urine cytology has high specificity but relatively low sensitivity for the diagnosis of urothelial neoplasia, particularly in low grade bladder tumors that by definition, lack high grade nuclear atypia and often show architectural patterns (papillary cluster formation) that overlap with the features associated with bladder lithiasis, inflammation, and other benign, reactive changes [36]. Several biomarkerbased urine tests have been approved by FDA for clinical use in bladder cancer [5, 7, 37], including ImmunoCyt, an assay that combines fluorescently labeled monoclonal antibodies for M344, LDQ10, 19A211, and antibodies to a glycosylated form of the carcinoembryonic antigen (CEA). Unfortunately, this test has a high false positive rate in samples from patients with microhematuria, cystitis or benign prostatic hyperplasia [12]. By contrast, the UroVysion test is a fluorescence in situ hybridization assay at detects aneuploidy of chromosome 3,7 , and 17 , as well as loss of the 9p21 locus. This test is sensitive for the detection of high-grade urothelial carcinomas but has a low sensitivity for the detection of low-grade urothelial carcinomas that generally lack of aneuploidy [7, 9, 11]. In our study, UroVysion testing was requested by Stony Brook Medicine urology clinical services for five cases that had negative 
cytologic findings and all were negative by both UroVysion and by K17 immunocytochemistry (data not shown). Although biopsy data was not available, none of these patients had a prior or subsequent diagnosis of urothelial carcinoma, suggesting that both K17 ICC and UroVysion provided accurate negative test results in this limited subset of cases. The diagnostic test performance of K17 compared to that of UroVysion, or any other biomarkers of urothelial carcinoma, however, remains to be established in future prospective clinical trials. Protein biomarker assays, including bladder tumor antigens (BTA-stat, BTA-TRAK), nuclear matrix protein-22 (BladderCheck and Bladder Cancer Test) and fibrinogen degradation products (ACCUDX), have also been FDA approved clinical use and have been found to enhance sensitivity but have lower specificity than cytology, especially in patients with inflammation and infection in the urinary tract $[4,5,13]$. Cxbladder is a gene expression test that detects and quantifies five mRNA biomarkers found in urine (IGFBP5, HOXA13, MDK, and CDK1 are associated with the growth and propagation of tumor tissue and the fifth biomarker (CXCR2) is a marker of inflammation to reduce false-positive results by identifying patients with non-malignant inflammatory conditions. These tests have a high sensitivity but lower specificity compared to urine cytology $[8,38]$. Thus, there remains an unmet need to identify and validate novel approaches for bladder cancer screening of urine cytology specimens.

Although our studies support the conclusion that high levels of K17 expression are seen in neoplastic lesions of the bladder mucosa, our urine cytology studies have thus far, only evaluated test performance in a limited number of cases of normal/benign/reactive changes or mild atypia vs. cases with a cytologic diagnosis of moderate atypia, severe atypia/suspicious for carcinoma, or positive for urothelial carcinoma. In this select set of cases, K17 immunocytochemistry was both more sensitive $(100 \%$ vs. $80 \%)$ and showed improved specificity ( $96 \%$ vs. $93 \%$ ), compared to cytology for the diagnosis of biopsy confirmed carcinoma. The sensitivity and specificity for K17 immunocytochemistry in a screening population, or to monitor for disease recurrence after therapeutic intervention still needs to be further investigated in a larger sample population. Recent data from the DETECT 1 study showed a prevalence of $6.9 \%$ for bladder cancer and $1.4 \%$ for upper urinary tract urothelial carcinoma among 556 patients from a multicenter prospective observational study of patients investigated for hematuria [39]. Thus based on our pilot data and extrapolating from data on disease prevalence in the DETEC 1 study, it would be necessary to test 1069 urine specimens to determine positive and negative predictive value in patients with hematuria with a test accuracy of $95 \%$.

In summary, K17 has the potential to serve as a sensitive and specific biomarker of urothelial neoplasia of the urinary bladder mucosa. Although the high sensitivity and specificity of the results in two patient cohorts suggests that the test could be a dependable adjunct for cancer surveillance, prospective clinical trials are still needed to define the potential role of $\mathrm{K} 17$ as a biomarker to enhance diagnostic accuracy in screening urine cytology.

Acknowledgements The Stony Brook Medicine BioBank provided tissue blocks for immunohistochemical studies.

Funding This work was supported by academic enrichment funds of the Department of Pathology at Stony Brook Medicine and KDx Diagnostic Inc.

\section{Compliance with ethical standards}

Conflict of interest N.W.K. and S.J. are employees of KDx Diagnostics Inc. and S.S.G. is a Chief Medical Officer of KDx. K.R.S., L.F. E.-H., N.W.K. are co-Inventors for Keratin 17 as a biomarker for bladder cancer (SBU Ref: R-8855-conversion deadline; US provisional Application No. 62/371,286; SSMP Docket: 33851P, International Publication number WO 2018/027091 A1). The remaining authors declare that they have no conflict of interest.

\section{References}

1. Siegel RL, Miller KD, Jemal A. Cancer statistics, 2018. CA Cancer J Clin. 2018;68:7-30.

2. Mariotto AB, Noone AM, Howlader N, et al. Cancer survival: an overview of measures, uses, and interpretation. J Natl Cancer Inst Monogr. 2014;2014:145-86.

3. Botteman MF, Pashos CL, Redaelli A, Laskin B, Hauser R. The health economics of bladder cancer: a comprehensive review of the published literature. Pharmacoeconomics. 2003;21: $1315-30$

4. van Rhijn BW, van der Poel HG, van der Kwast TH. Urine markers for bladder cancer surveillance: a systematic review. Eur Urol. 2005;47:736-48.

5. Eissa S, Swellam M, Sadek M, et al. Comparative evaluation of the nuclear matrix protein, fibronectin, urinary bladder cancer antigen and voided urine cytology in the detection of bladder tumors. J Urol. 2002;168:465-9.

6. Bosschieter J, Lutz C, Segerink LI, et al. The diagnostic accuracy of methylation markers in urine for the detection of bladder cancer: a systematic review. Epigenomics. 2018;10:673-687.

7. Dimashkieh H, Wolff DJ, Smith TM, et al. Evaluation of urovysion and cytology for bladder cancer detection: a study of 1835 paired urine samples with clinical and histologic correlation. Cancer Cytopathol. 2013;121:591-7.

8. Breen V, Kasabov N, Kamat AM, et al. A holistic comparative analysis of diagnostic tests for urothelial carcinoma: a study of Cxbladder Detect, UroVysion(R) FISH, NMP22(R) and cytology based on imputation of multiple datasets. BMC Med Res Methodol. 2015;15:45.

9. Zellweger T, Benz G, Cathomas G, et al. Multi-target fluorescence in situ hybridization in bladder washings for prediction of recurrent bladder cancer. Int J Cancer. 2006;119:1660-5.

10. Lavery HJ, Zaharieva B, McFaddin A, Heerema N, Pohar KS. A prospective comparison of UroVysion FISH and urine cytology in bladder cancer detection. BMC Cancer. 2017;17:247.

11. Bollmann M, Heller H, Bankfalvi A, Griefingholt H, Bollmann R. Quantitative molecular urinary cytology by fluorescence in situ 
hybridization: a tool for tailoring surveillance of patients with superficial bladder cancer? BJU Int. 2005;95:1219-25.

12. Comploj E, Mian C, Ambrosini-Spaltro A, et al. uCyt+/ImmunoCyt and cytology in the detection of urothelial carcinoma: an update on 7422 analyses. Cancer Cytopathol. 2013;121:392-7.

13. Choi HS, Lee SI, Kim DJ, Jeong TY. Usefulness of the NMP22BladderChek Test for Screening and Follow-up of Bladder Cancer. Korean J Urol. 2010;51:88-93.

14. Cheung G, Sahai A, Billia M, Dasgupta P, Khan MS. Recent advances in the diagnosis and treatment of bladder cancer. BMC Med. 2013;11:13.

15. Escobar-Hoyos LF, Yang J, Zhu J, et al. Keratin 17 in premalignant and malignant squamous lesions of the cervix: proteomic discovery and immunohistochemical validation as a diagnostic and prognostic biomarker. Mod Pathol. 2014;27:621-30.

16. Escobar-Hoyos LF, Shah R, Roa-Pena L, et al. Keratin-17 promotes p27KIP1 nuclear export and degradation and offers potential prognostic utility. Cancer Res. 2015;75:3650-62.

17. Mockler D, Escobar-Hoyos LF, Akalin A, et al. Keratin 17 Is a prognostic biomarker in endocervical glandular neoplasia. Am J Clin Pathol. 2017;148:264-73.

18. Merkin RD, Vanner EA, Romeiser JL, et al. Keratin 17 is overexpressed and predicts poor survival in estrogen receptor-negative/human epidermal growth factor receptor-2-negative breast cancer. Hum Pathol. 2017;62:23-32.

19. Regenbogen E, Mo M, Romeiser J, et al. Elevated expression of keratin 17 in oropharyngeal squamous cell carcinoma is associated with decreased survival. Head N eck. 2018;40:1788-98.

20. Chivu-Economescu M, Dragu DL, Necula LG, et al. Knockdown of KRT17 by siRNA induces antitumoral effects on gastric cancer cells. Gastric Cancer. 2017;20:948-59.

21. Wang YF, Lang HY, Yuan J, et al. Overexpression of keratin 17 is associated with poor prognosis in epithelial ovarian cancer. Tumour Biol. 2013;34:1685-9.

22. Rhodes DR, Yu J, Shanker K, et al. ONCOMINE: a cancer microarray database and integrated data-mining platform. Neoplasia. 2004;6:1-6.

23. Hodis E, Watson IR, Kryukov GV, et al. A landscape of driver mutations in melanoma. Cell. 2012;150:251-63.

24. Ruopp MD, Perkins NJ, Whitcomb BW, Schisterman EF. Youden Index and Optimal Cut-Point Estimated from Observations Affected by a Lower Limit of Detection. Biom J Biom Z. 2008;50:419-30.

25. Moll R, Franke WW, Schiller DL, Geiger B, Krepler R. The catalog of human cytokeratins: patterns of expression in normal epithelia, tumors and cultured cells. Cell. 1982;31:11-24.
26. Chu PG, Weiss LM. Keratin expression in human tissues and neoplasms. Histopathology. 2002;40:403-39.

27. Maxwell JP, Wang C, Wiebe N, Yilmaz A, Trpkov K. Long-term outcome of primary Papillary Urothelial Neoplasm of Low Malignant Potential (PUNLMP) including PUNLMP with inverted growth. Diagn Pathol. 2015;10:3.

28. Kolokythas A, Schwartz JL, Pytynia KB, et al. Analysis of RNA from brush cytology detects changes in B2M, CYP1B1 and KRT17 levels with OSCC in tobacco users. Oral Oncol. 2011;47:532-6.

29. Somji S, Cao L, Mehus A, et al. Comparison of expression patterns of keratin $6,7,16,17$, and 19 within multiple independent isolates of $\mathrm{As}(+3)$ - and $\mathrm{Cd}(+2)$-induced bladder cancer: keratin 6, 7, 16, 17, and 19 in bladder cancer. Cell Biol Toxicol. 2011;27:381-96.

30. Guelstein VI, Tchipysheva TA, Ermilova VD, Troyanovsky SM. Immunohistochemical localization of cytokeratin 17 in transitional cell carcinomas of the human urinary tract. Virchows Arch B Cell Pathol Incl Mol Pathol. 1993;64:1-5.

31. Lopez-Beltran A, Montironi R, Vidal A, Scarpelli M, Cheng L. Urothelial dysplasia of the bladder: diagnostic features and clinical significance. Anal Quant Cytopathol Histopathol. 2013;35: 121-9.

32. Comperat E, Camparo P, Haus R, et al. Immunohistochemical expression ofp63, p53 and MIB-1 in urinary bladder carcinoma. A tissue microarray study of 158 cases. Virchows Arch. 2006;448:319-24.

33. Arias-Stella JA 3rd, Shah AB, Gupta NS, Williamson SR. CK20 and p53 Immunohistochemical Staining Patterns in Urinary Bladder Specimens With Equivocal Atypia. Arch Pathol Lab Med. 2018;142:64-9.

34. Wu C-T, Lin W-Y, Chang Y-H, Chen W-C, Chen M-F. Impact of CD44 expression on radiation response for bladder cancer. $\mathrm{J}$ Cancer. 2017;8:1137-44.

35. Reuter VE. The pathology of bladder cancer. Urology. 2006;67:11-7. discussion7-8

36. Wiener HG, Vooijs GP, van't Hof-Grootenboer B. Accuracy of urinary cytology in the diagnosis of primary and recurrent bladder cancer. Acta Cytol. 1993;37:163-9.

37. Weinstein JN, Akbani R, Broom BM et al. Cancer Genome Atlas Research Network. Comprehensive molecular characterization of urothelial bladder carcinoma. Nature. 2014;507:315-22.

38. Darling D, Luxmanan C, O'Sullivan P, Lough T, Suttie J. Clinical utility of cxbladder for the diagnosis of urothelial carcinoma. Adv Ther. 2017;34:1087-96.

39. Tan WS, Sarpong R, Khetrapal P, et al. Does urinary cytology have a role in haematuria investigations? BJU Int. 2018. 